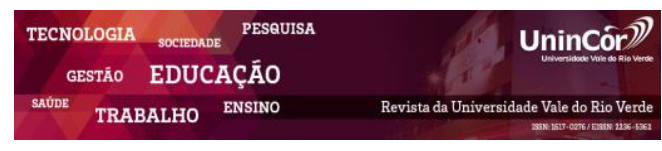

Revista da Universidade Vale do Rio Verde ISSN: 1517-0276 / EISSN: 2236-5362 v. $16 \mid \mathbf{n}^{\circ} .3$ | Ano 2018

Elaine Aparecida Rocha Domingues Universidade Vale do Rio Verde (UNINCOR) elainerocha.contato@gmail.com

Camila Regina de Souza Antenor Universidade Vale do Rio Verde (UNINCOR) kamilinha-kaka@hotmail.com

Maiúme Roana Ferreira de Carvalho Universidade Vale do Rio Verde (UNINCOR) enf_maiume@yahoo.com.br

Aline Ferreira Souza de Carvalho Universidade Vale do Rio Verde (UNINCOR) aline.carvalho@gmail.com

\section{BEM-ESTAR ESPIRITUAL DO PROFISSIONAL DE ENFERMAGEM NO AMBIENTE HOSPITALAR}

\section{RESUMO}

Espiritualidade e religiosidade constituem as duas das mais comuns e antigas formas que a humanidade encontrou de dar significado para a vida, bem como oferecer às pessoas meios para enfrentar às condições adversas. Objetivou-se avaliar as percepções do profissional de enfermagem frente à espiritualidade e ao cuidado espiritual durante a assistência. Trata-se de uma pesquisa quantitativa, descritiva e transversal, realizada com 32 profissionais de enfermagem, de um hospital particular de Minas Gerais, avaliados no período de agosto a setembro de 2018. Foram utilizados três instrumentos: o questionário sociodemográfico, a Escala de espiritualidade Spirituality Self Rating Scale e a Escala de Bem-estar Espiritual (EBE). Os dados coletados foram agrupados em um banco de dados e posteriormente avaliados para análise descritiva e apresentados em forma de gráficos e tabelas. A média de idade dos participantes foi de 34 anos, permeando o intervalo de 31-40 anos. Predominou-se o gênero feminino com ensino superior completo, sendo a média de tempo de serviço de sete anos, variando de 6-10 anos. Em relação à espiritualidade e religiosidade, $87,50 \%$ sabem a distinção entre elas. A maioria dos profissionais de enfermagem apresentou alto índice de bem-estar espiritual (78\%) e de espiritualidade (81\%), bem como reconhecem a importância da espiritualidade na saúde. Conclui-se que profissionais de enfermagem possuem alta percepção de espiritualidade e religiosidade. Além disso, acreditam na importância do cuidado espiritual durante a assistência.

Palavras-chave: Bem-Estar Espiritual. Enfermagem. Cuidado Espiritual. Espiritualidade. Religiosidade.

\section{SPIRITUAL WELL-BEING OF THE NURSING PROFESSIONAL IN THE HOSPITAL ENVIRONMENT}


of seven years of service, varying from 6-10 years. In relation to spirituality and religiosity, $87.50 \%$ know the distinction between them. The majority of the participants had a high rate of spiritual well-being $(78 \%)$ and spirituality $(81 \%)$ and recognize the importance of spirituality in health. It is concluded that nursing professionals have a high perception of spirituality and religiosity. Also, they believe in the importance of spiritual care during assistance.

Keywords: Spiritual Well-Being. Nursing. Spiritual Care. Spirituality. Religiosity.

\section{Recebido em: 24/08/2018 - Aprovado em: 09/11/2018 - Disponibilizado em: 30/12/2018}

\section{INTRODUÇÃO}

O binômio espiritualidade e religiosidade constituem as duas das mais comuns e antigas formas a qual a humanidade encontrou de dar significado para a vida, bem como permitir aos indivíduos meios para enfrentar as condições adversas (CAYANA et al., 2017).

A espiritualidade pode ser definida como uma inclinação humana na busca do significado para a vida, por meios de conceitos que vão além do que se pode tocar: um sentido de conexão com algo que é maior que si próprio, que pode ou não incluir uma participação religiosa formal, tem como referência questões sobre os significados do propósito da vida, tendo a crença de aspecto espiritualista para justificar a existência humana (OLIVIEIRA; FRAZILI, 2017).

Por outro lado, a religião deriva do latim religare que significa a ligação de valores éticos entre Deus e os homens. O termo religião diz respeito ao lado social organizado em sistemas de crenças, valores e ritos religiosos ao qual uma pessoa, na prática da sua religiosidade, pode ou não aderir formalmente. Já o sentido de religiosidade seria o sentimento que motiva e impulsiona o sujeito a vivenciar fenômenos religiosos. Ressalta-se que a religião admira um ser superior que se apresenta de diversas formas, sendo elas históricas, étnicas, temporais e culturais. Por outro lado, a religiosidade se manifesta por meio da percepção emocional, religiosa e particular de cada sujeito (NUNES, 2017).

Existe diferença entre religiosidade e espiritualidade. A espiritualidade é um recurso individual e subjetivo de conexão consigo mesmo, com o outro, com Deus ou outra divindade. A religiosidade, por sua vez, pode ser entendida como a prática de um sistema organizado de crenças (religião), que abrange a presença de hierarquias, líderes/sacerdotes e doutrinas a serem seguidas; é o quanto um indivíduo acredita, segue e pratica essa religião (OLIVEIRA; SANTOS; YARID, 2018).

$\mathrm{O}$ bem-estar espiritual no momento atual tem sido o centro de diversas investigações, sobretudo associadas com os processos de saúde, instituindo-se como um estado psicológico positivo. Vários autores enfatizam a importância deste constructo na reestruturação das 
expectativas positivas da vida diante da adversidade. $\mathrm{O}$ bem-estar espiritual é uma vivencia de apoio e fortalecimento, à qual a pessoa utiliza de forma proposital em uma circunstância de crise, de forma a coadjuvar a resolução da mesma (SILVA, 2017).

No contexto da saúde, a espiritualidade tem se tornado um padrão a ser estabelecido na prática médica diária. A doença permanece como essência de grande efeito sobre aspectos de abordagem, desde a fisiopatologia básica até sua complexa relação social, psíquica e econômica. É fundamental admitir que esses diversos aspectos estão interligados em múltipla interação (GIOVELLI et al., 2008).

Reginato; Benedetto e Gallian (2016) enfatizam que a identificação dos 'primeiros médicos' se confunde, na História, com a figura de sacerdotes, xamãs e curandeiros, e isto fica claro quando verificamos que os males do corpo eram principalmente relacionados à ação negativa de deuses como meio de punição, e situações místicas incompreensíveis no mundo natural.

Mesmo com o desenvolvimento da ciência, ainda se manteve uma ligação entre a cura do corpo e a condição de crença do paciente em um campo sobrenatural em que, mediante a sua fé ou a interposição de orações e cultos, o paciente poderia encontrar a cura, principalmente quando esgotados todos os recursos conhecidos (REGINATO; BENEDE-TTO; GALLIAN, 2016).

A espiritualidade tem sido bastante examinada no que se refere à sua ligação com a saúde humana. A Organização Mundial de Saúde (OMS) tem observado minuciosamente indagações sobre a espiritualidade enquanto parte integrante do conceito multidimensional de saúde. Assim como as dimensões físicas, psicológicas e sociais, o bem-estar espiritual é encarado como uma dimensão do estado de saúde (MOREIRA; PEREIRA, 2016).

A conexão histórica entre espiritualidade e enfermagem é antiga (MOREIRA; PEREIRA, 2016). A enfermagem sempre teve um legado fortemente holístico e os enfermeiros a tem praticado com sensibilidade para as necessidades físicas, psicossociais e espirituais das pessoas. Apesar de nem todos os enfermeiros considerarem a assistência espiritual primordial em sua rotina de trabalho, essa dimensão é muito importante para o cuidado (ABDALA et al.,2017).

Quando se traça um perfil histórico da profissão de enfermagem sabe-se que a mesma se originou praticamente da igreja, quando as Irmãs de Caridade da ordem São Vicente de Paula em 1617 começaram a reunir outras irmãs católicas para servir tanto a hospitais religiosos quanto seculares. Em 1837, ao receber "um chamado de Deus", Florence Nightingale buscou treinamento entre as Irmãs de Caridade e Diaconisas protestantes, começando a colocar em prática os princípios que ela havia aprendido (ABDALA et al., 2017).

Todavia, apesar da conotação religiosa que cerca $\mathrm{o}$ ato de cuidar desde a sua origem, somente a partir do início da produção científica em enfermagem nasceu uma nova visão, pautada na identificação das necessidades espirituais, independente da pessoa ser ou não religioso (ABDALA et al., 2017). 
O tema espiritualidade ainda é pouco explorado no âmbito acadêmico. Fato esse preocupante, pois os profissionais, primordialmente os da saúde, necessitam entender sobre a temática para prestar uma assistência integral ao paciente, favorecendo a sua recuperação.

Atrelado a este fato, existem diversos artigos na literatura que abordam a espiritualidade sobre a ótica dos pacientes, enquanto que há escassas pesquisas que referem a percepção dos profissionais sobre a espiritualidade no cuidado. Vale ressaltar que o profissional para oferecer um tratamento espiritual de qualidade, deve estar capacitado para prestar este tipo de cuidado.

Portanto, objetivou-se avaliar as percepções do profissional de enfermagem frente à espiritualidade e ao cuidado espiritual durante a assistência.

\section{MATERIAL E MÉTODOS}

Trata-se de uma pesquisa quantitativa, descritiva e transversal. O cenário de estudo foi o Hospital Humanitas Unimed, que conta com 6 alas subdivididas em ala I (12 leitos), ala II (35 leitos), ala III (26 leitos), CTI (8 leitos), Centro Cirúrgico (7 salas) Prontomed (12 leitos). (UNIMED VARGINHA, 2018).

A população do estudo foi composta por 32 profissionais de enfermagem do Hospital Humanitas Unimed, avaliados no período de agosto a setembro de 2018, alocados no mesmo local de trabalho. Para inclusão do sujeito de pesquisa no estudo, os seguintes critérios de elegibilidade foram considerados: serem da categoria de enfermagem registrados no Coren (Conselho Regional de Enfermagem), de ambos os sexos e não estarem no período de férias ou em afastamento por saúde e/ou licença maternidade.

Para a coleta de dados, foi utilizado o questionário sociodemográfico, a Escala de espiritualidade "Spirituality Self Rating Scale" e a Escala de Bem-estar Espiritual (EBE).

Os dados sociodemográficos avaliados foram: sexo, idade, estado civil, renda familiar, escolaridade e tempo de função. Também foram investigadas: religião, prática religiosa e frequência da atividade religiosa. A prática religiosa foi investigada por meio da pergunta "Você pratica atividade religiosa (faz orações, preces, meditação, leitura de textos espirituais...)? ", com opção dicotômica (sim e não). Também foram investigadas questões referente à espiritualidade e religiosidade, por meio das indagações: "Você sabe a diferença de espiritualidade e religiosidade?" "Você acha que a espiritualidade influencia no cuidado? Sim? Não? Por quê? ".

A EBE foi elaborada por Paloutzian; Elisson em 1982 e foi adaptada e validada para o Brasil por Marques; Sarriera e Dell'Aglio em 2009, cuja consistência interna foi de 0,92, demonstrando alta fidedignidade. A EBE possui 20 itens divididos em duas dimensões: o bemestar existencial (BEE, composta por 10 itens, uma dimensão "horizontal", associada a um propósito na vida e satisfação com a própria existência) e o bem-estar religioso (BER, também composta por 10 itens, uma dimensão "vertical", referente a um senso de bem-estar em relação a Deus). Os itens são apresentados em 
escala Likert de seis opções: Concordo Totalmente (CT), concordo mais que discordo (Cd), Concordo Parcialmente (CP), Discordo Parcialmente (DP), discordo mais que concordo (Dc), e Discordo Totalmente (DT). As questões com conotação positiva $(3,4,7,8,10,11,14,15$, 17, 19 e 20) têm sua pontuação somada da seguinte maneira, $\mathrm{CT}=6, \mathrm{Cd}=5, \mathrm{CP}=4, \mathrm{DP}=3$, $\mathrm{Dc}=2$ e DT=1. As demais questões são negativas e devem ser somadas de forma invertida $(\mathrm{CT}=1$, $\mathrm{Cd}=2, \mathrm{CP}=3$ e assim por diante). Assim, os escores da dimensão BER são obtidos da soma das pontuações dos itens ímpares da escala, e os escores da dimensão BEE são obtidos da soma dos itens pares (a amplitude é de 6 a 60 pontos). Para o escore geral de bem-estar espiritual, os pontos equivalem aos intervalos de 20 a 40, 41 a 99 e 100 a 120 e, nas duas subescalas, os intervalos são 10 a 20, 21 a 49 e 50 a 60 pontos, para baixo, moderado e alto bem-estar espiritual, respectivamente. Os resultados da EBE são considerados positivos para escore alto, e negativo para moderado e baixo (VOLCAN et al, 2003; GASTAUD et al, 2006).

A escala de espiritualidade denominada "Spirituality Self Rating Scale", é um dispositivo de autopreenchimento formado por seis itens que classificam aspectos da espiritualidade do indivíduo, para sua utilização. É fundamental fazer o somatório de pontos, que varia de 6 a 30 . Deve-se, previamente, recodificar cada item do instrumento (por exemplo, escore de 5 torna-se 1; 2 torna-se 4; e assim por diante). As respostas recodificadas são somadas para elaborar o escore total, e este, por sua vez, representa o nível de orientação espiritual.
Os dados coletados foram agrupados em um banco de dados usando uma planilha eletrônica e posteriormente avaliada para análise descritiva. Os resultados foram apresentados em forma de gráficos e tabelas, utilizadas medidas de posição (média, desvio padrão) para as variáveis consideradas contínuas e frequência relativa e absoluta para as variáveis categóricas.

A pesquisa respeitou a Resolução ${ }^{\circ} 466$, de 12 de dezembro de 2012, considerando o respeito pela dignidade humana e pela especial proteção devida aos participantes das pesquisas científicas envolvendo seres humanos (CONSELHO NACIONAL DE SAÚDE, 2013). Além disso, foi aprovada pelo Comitê de Ética em Pesquisa da UninCor, sob o parecer consubstanciado $\mathrm{n}^{\mathrm{o}}$ 2.857.506. Obteve-se o consentimento dos participantes por meio da assinatura do Termo de Consentimento Livre e Esclarecido.

\section{RESULTADOS E DISCUSSÃO}

A média de idade foi de 34 anos, permeando o intervalo de 31-40 anos $(53,13 \%)$, predominou o gênero feminino com 78,13\%, prevalecendo o nível de escolaridade de ensino superior completo $(50 \%$,) sendo a média de tempo de serviço de sete anos, variando de 6-10 anos $(43,75 \%)$ (Tabela 1$)$.

Do total de entrevistados, $71,88 \%$ declararam-se praticantes religiosos, sendo que destes, 65,63\%, eram católicos (Gráfico 1). 
Tabela 1 - Características sóciodemográficas dos profissionais de enfermagem do Hospital Humanitas, Varginha, Minas Gerais ( $\mathrm{n}=32)$.

\begin{tabular}{|c|c|c|}
\hline Variável & $\begin{array}{l}\text { Frequência } \\
\text { absoluta }\end{array}$ & $\begin{array}{l}\text { Frequência } \\
\text { relativa }\end{array}$ \\
\hline \multicolumn{3}{|l|}{ Gênero } \\
\hline Masculino & 7 & 21,88 \\
\hline Feminino & 25 & 78,13 \\
\hline \multicolumn{3}{|l|}{ Idade } \\
\hline $20-30$ & 10 & 31,25 \\
\hline $31-40$ & 17 & 53,13 \\
\hline $41-50$ & 5 & 15,63 \\
\hline \multicolumn{3}{|l|}{ Escolaridade } \\
\hline Médio completo & 11 & 34,38 \\
\hline Superior incompleto & 5 & 15,63 \\
\hline Superior completo & 16 & 50,00 \\
\hline \multicolumn{3}{|l|}{ Tempo de serviço } \\
\hline $1-5$ anos & 11 & 34,38 \\
\hline 6-10 anos & 14 & 43,75 \\
\hline $11-15$ anos & 3 & 9,38 \\
\hline $16-20$ anos & 1 & 3,13 \\
\hline 21-30 anos & 3 & 9,38 \\
\hline
\end{tabular}

Gráfico 1 - Religião dos profissionais de enfermagem do Hospital Humanitas, Varginha, Minas Gerais ( $\mathrm{n}=$ 32).

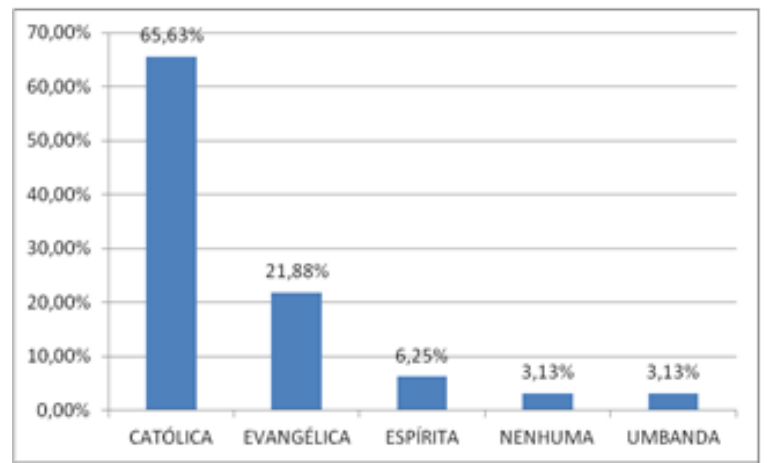

Quando questionados sobre espiritualidade e religiosidade, $87,50 \%$ sabem a diferenciação entre elas. Além disso, 90,63\% acreditam que a espiritualidade de alguma maneira pode influenciar no cuidado (Tabela 2).

Os dados referentes à determinação do bem-estar espiritual estão representados na Tabela 3 . Observa-se que $78 \%$ dos profissionais de enfermagem apresentaram um alto índice de bem-estar espiritual. No que se refere ao bemestar religioso (BER), 63\% dos entrevistados apresentaram alto índice. Em relação ao bemestar existencial (BEE), $72 \%$ da população apresentou alto índice.

Tabela 2 - Investigação pessoal do bem-estar espiritual dos profissionais de enfermagem do Hospital Humanitas, Varginha, Minas Gerais $(n=32)$.

\begin{tabular}{lcc}
\hline Variável & $\begin{array}{c}\text { Frequência } \\
\text { absoluta }\end{array}$ & $\begin{array}{c}\text { Frequência } \\
\text { relativa }\end{array}$ \\
\hline Prática religiosa? & & \\
\hline Sim & 23 & 71,88 \\
Não & 9 & 28,13
\end{tabular}

\begin{tabular}{lcc}
\hline \multicolumn{3}{l}{ Diferença espiritualidade/religiosidade? } \\
\hline Sim & 28 & 87,50 \\
Não & 4 & 12,50
\end{tabular}

Espiritualidade influencia no cuidado?

\begin{tabular}{lcc}
\hline Sim & 29 & 90,63 \\
Não & 3 & 9,38 \\
\hline
\end{tabular}

Tabela 3 - Bem-estar espiritual dos profissionais de enfermagem do Hospital Humanitas, Varginha, Minas Gerais $(n=32)$.

\begin{tabular}{lcccc}
\hline Variável & $\begin{array}{c}\text { FREQ. } \\
\text { ABSOLUTA }\end{array}$ & $\begin{array}{c}\text { FREQ. } \\
\text { RELATIVA }\end{array}$ & Média & DP \\
\hline EBE total & & & 107,0 & 9,3 \\
\hline $\begin{array}{l}\text { EBE }^{1} \\
\text { moderado }\end{array}$ & 7 & 22 & & \\
$\begin{array}{l}\text { EBE alto } \\
\text { Total }\end{array}$ & 25 & 78 & & \\
\hline BER total & 32 & 100 & & \\
\hline BER $^{2}$ & 12 & 38 & & \\
rmoderado & 20 & 63 & & \\
BER alto & 32 & 100 & & \\
Total & 1 & & 50,4 & 7,1 \\
\hline BEE total & & 3 & & 3,5 \\
\hline $\begin{array}{l}\text { BEE } \\
\text { moderado }\end{array}$ & 23 & 72 & & \\
BEE alto & 24 & 75 & & \\
Total & 23 & & & \\
\hline
\end{tabular}

${ }^{1}$ EBE: bem-estar espiritual; ${ }^{2}$ BER: bem-estar religioso; ${ }^{3}$ BEE: bem-estar existencial.

Em relação à espiritualidade determinada pela escala "Spirituality Self Rating Scale", $81 \%$ dos profissionais de enfermagem 
apresentaram alta espiritualidade e $19 \%$ espiritualidade moderada (Tabela 4).

Tabela 4 - Espiritualidade dos profissionais de enfermagem do Hospital Humanitas, Varginha, Minas Gerais $(n=32)$.

\begin{tabular}{lcccc}
\hline & $\begin{array}{c}\text { Freq. } \\
\text { relativa }\end{array}$ & $\begin{array}{c}\text { Freq. } \\
\text { absoluta }\end{array}$ & Média & DP \\
\hline SSRS ${ }^{1}$ total & & & 24,6 & 3,4 \\
$\begin{array}{l}\text { Espiritualidade } \\
\text { moderada }\end{array}$ & 6 & 19 & & \\
$\begin{array}{l}\text { Espiritualidade } \\
\text { alta }\end{array}$ & 26 & 81 & & \\
Total & 32 & 100 & & \\
& & &
\end{tabular}

${ }^{1}$ SSRS: Escala de espiritualidade "Spirituality Self Rating Scale".

No presente estudo verificou-se que a maioria dos participantes da pesquisa é do gênero feminino, o que corrobora com dados nacionais que apontam este gênero como maioria entre profissionais de saúde, sobretudo a equipe de enfermagem em unidades hospitalares (CONSELHO FEDERAL DE ENFERMAGEM, 2015).

Em relação à escolaridade, predominou o ensino superior completo, com tempo médio de serviço de sete anos, diferente do observado no Hospital Universitário da cidade de São Paulo, onde a média foi de 10,5 anos (PENHA; SILVA, 2012).

A religião predominante foi a católica, o que está de acordo com dados nacionais que demostram que a maior parte da população brasileira pertence a esta religião (IBGE, 2010). Quando utilizado o método EBE, o presente estudo revelou a presença de escores positivos na escala de bem-estar espiritual, existencial e religioso. Tal cenário foi similar ao estudo realizado no Hospital Israelita Albert Einstein, onde se aplicou o mesmo método de avaliação (PEDRÃO; BERESIN, 2010).
Em relação à espiritualidade, pode-se observar que os profissionais de enfermagem apresentam um elevado índice de espiritualidade. De maneira semelhante, quando aplicada em pacientes nas Comunidades Terapêuticas do estado de São Paulo, esta mesma metodologia indicou um grau elevado de espiritualidade. A versão original desta escala tem sido também utilizada para pacientes, médicos e demais profissionais de saúde (GONÇALVES; PILLON, 2009).

A maioria soube diferenciar espiritualidade de religiosidade, diferente do estudo realizado no Hospital-escola Estadual do Interior Paulista (NASCIMENTO et al.,2013). Além disso, a maioria da população acredita que a espiritualidade influencia no cuidado ao paciente. Corroborando com estes resultados, outros estudos demostram esta mesma percepção, onde acredita-se que a espiritualidade afeta positivamente na saúde dos pacientes e na assistência prestada pelo profissional de enfermagem (GONÇALVES; PILLON, 2009; TOMASSO; BEL-TRAME; LUCCHETTI, 2011).

A espiritualidade tem sido amplamente abordada e discutida em eventos internacionais de enfermagem. A questão da espiritualidade, no que se alude ao profissional da saúde, está ligada, particularmente, à compaixão, que significa tentar compreender o outro, ter empatia, sensibilidade diante do sofrimento alheio como se fosse seu próprio sofrimento A espiritualidade se manifesta nas ações e na correlação entre as atitudes e pensamentos; está na (com) paixão pelo ser humano e assim, no cuidar do outro como cuidaríamos de nós mesmos. A 
espiritualidade para o ser humano traz a ele a energia para o enfrentamento da vida e busca de significado para toda a sua existência. Assim, pode-se inferir que o ser humano, quando perde sua espiritualidade, perde a vontade de viver e de lutar pela vida (SÁ, 2009).

O enfermeiro tem a função de auxiliar o paciente e sua família a ligar-se com o que lhe dá força para persistir lutando. Diante disso, é importante que cada profissional conheça a sua própria linguagem espiritual, suposições e experiências e, assim, avaliem a necessidade de intervenção neste campo durante a assistência ao paciente. Enfermeiros conscientes de sua religiosidade e espiritualidade podem proporcionar melhor cuidado, uma vez que, se tornam mais sensíveis e capazes de entrar em um diálogo mais profundo com o paciente (NASCIMENTO, 2010).

Diante dos resultados observados na presente pesquisa, pode-se evidenciar a necessidade de preparar e esclarecer o profissional de enfermagem para a prestação de cuidado espiritual. Assim, como observado e destacado também por outros autores, é cada vez mais necessária à realização de mais pesquisas que abordem esta temática (VAN LEEWEN; TIESINGA; POST, 2006; PAULA;NASCIMENTO; ROCHA, 2009).

Neste contexto, espera-se que novas pesquisas possam vir a ser desenvolvidas considerando, por exemplo, uma investigação sore os motivos pelos quais muitos profissionais ainda desacreditam que a espiritualidade influencia ou não no cuidado. Além disso, sugere-se a continuidade das pesquisas em hospitais particulares e sua abrangência para o
Sistema Único de Saúde (SUS). Com isso, é possível realizar um estudo comparativo e estabelecer se o conhecimento e níveis de espiritualidade dos profissionais do âmbito privado diferem dos profissionais do SUS e, com isso, avaliar se o ambiente de trabalho exerce influência sobre esses profissionais.

\section{CONCLUSÃO}

Conclui-se que os profissionais de enfermagem possuem alta percepção de espiritualidade e religiosidade. Além disso, acreditam na importância da espiritualidade para o cuidado. Entretanto, vale ressaltar que o índice observado ainda não é o ideal, uma vez que, se trata de profissionais que lidam com seres humanos. Em função disso, a não percepção da espiritualidade e o baixo índice de espiritualidade de alguns profissionais podem interferir na prestação do cuidado.

\section{REFERÊNCIAS}

ABDALA, G. A. et al. Religião, Espiritualidade e a Enfermagem. Revista Família, Ciclos de Vida e Saúde no Contexto Social, v. 5, p. 154-164, 2017.

CAYANA, E. G. et al. Religiosidade e Espiritualidade no Enfrentamento do Câncer: Uma Revisão Narrativa de Estudos Qualitativos. CIAIQ, v. 2, 2017.

Conselho Federal de Enfermagem. Perfil da Enfermagem na Bahia. 2015. Disponível em: http://www.cofen.gov.br/cofen-lanca-perfil-daenfermagem-na-bahia 31985.html. Acesso em:14/11/2018.

Conselho Nacional de Saúde. Publicada resolução 466 do CNS que trata de pesquisas em seres humanos e atualiza a resolução 196.2013. Disponível em: http://conselho.saude.gov.br/ultimas_noticias/2013/06 jun 14 publicada_resolucao.html. Acesso em:02/12/2018.

GASTAUD, M. B. et al. Bem-estar espiritual e transtornos psiquiátricos menores em estudantes de 
psicologia: estudo transversal. Rev Psiquiatr Rio Gd Sul, v. 28, n. 1, p. 12-8, 2006.

GIOVELLI, G. et al. Espiritualidade e religiosidade: uma questão bioética. Rev. Sorbi, v. 1, n. 5, p. 1-12, 2008.

GONÇALVES, A. M. de S.; PILLON, S. C.. Adaptação transcultural e avaliação da consistência interna da versão em português da Spirituality Self Rating Scale (SSRS). Revista de Psiquiatria Clínica, v. 36, n. 1, p. 10-15, 2009.

IBGE. Instituto Brasileiro de Geografia e Estatística. Censo Demográfico 2010. Disponível em: http://www.ibge.gov.br/home/estatistica/populacao/ce nso2010/caracteristicas_religiao_deficiencia/caracteris ticas_religiao_deficiencia_tab_pdf.sht Acesso em:14/11/2018.

MARQUES, L. F.; SARRIERA, J. C; DELL'AGLIO, D. D. Adaptação e validação da Escala de Bemestar Espiritual (EBE). Avaliação psicológica, v. 8, n. 2, 2009.

MOREIRA, C; PEREIRA, S. Impacto da Espiritualidade na Saúde Física. 2016. Trabalho de Conclusão de Curso.

NASCIMENTO, L. C. et al. Spirituality and religiosity in the perspectives of nurses. Texto $\&$ Contexto-Enfermagem, v. 22, n. 1, p. 52-60, 2013.

NUNES, L. C. Morte e religiosidade/espiritualidade no contexto hospitalar: o que dizem as pesquisas realizadas com profissionais? 2017. $49 \mathrm{f}$.

Monografia (Graduação em Psicologia) -

Universidade Católica de Brasília, Brasília, 2017.

OLIVEIRA, R. M.; S, ROSE, M. M.; YARID, S. D. Espiritualidade/religiosidade e o humanizaSUS em Unidades de Saúde da Família. Revista Brasileira em Promoção da Saúde, v. 31, n. 1, 2018.

OLIVERIA, F. F; FRAZILI, R. T. V. Espiritualidade: seu significado no contexto do acadêmico de enfermagem. Revista Eletrônica de Enfermagem do Vale do Paraíba, n. 02, 2017.

PAULA, E. S.; NASCIMENTO, L. C.; ROCHA, S. M. M. Religião e espiritualidade: experiência de famílias de crianças com Insuficiência Renal Crônica. Revista Brasileira de Enfermagem, v. 62, n. 1, p. 100-106, 2009.

PEDRÃO, R. B.; BERESIN, R.. O enfermeiro frente à questão da espiritualidade. Rev Einstein, v. 8, p. 86$91,2010$.

PENHA, R. M. et al. Significado de espiritualidade para a enfermagem em cuidados intensivos. Texto $\&$ Contexto-Enfermagem, v. 21, n. 2, p. 260-268, 2012.
REGINATO, V.; BENEDETTO, M. A. C.;

GALLIAN, D. M. C. Espiritualidade e saúde: uma experiência na graduação em medicina e enfermagem. Trabalho, Educação e Saúde, v. 14, n. 1, p. 237-255, 2016.

SÁ, A. C. Reflexão sobre o cuidar em Enfermagem: uma visão do ponto de vista da espiritualidade humana e da atitude crística. O mundo da saúde, v. 33, n. 2, p. 205-217, 2009.

SILVA, J. R. M. de A. Bem-estar Espiritual e Saúde (Mental) de Estudantes Universitários. 2017. Tese de Doutorado.

TOMASSO, C. S.; BELTRAME, I. L.; LUCCHETTI, G. Conhecimentos e atitudes de docentes e alunos em enfermagem na interface espiritualidade, religiosidade e saúde. Revista Latino-Americana de

Enfermagem, v. 19, n. 5, 2011.

UNIMED VARGINHA. Sobre o hospital. Disponível em:<https://www.unimed.coop.br/web/varginha/hospit al/sobre-o-hospital> Acesso em: 20 maio 2018.

VAN LEEUWEN, R. et al. Spiritual care: implications for nurses' professional responsibility. Journal of clinical nursing, v. 15, n. 7, p. 875-884, 2006.

VOLCAN, S. M. A. et al. Relação entre bem-estar espiritual e transtornos psiquiátricos menores: estudo transversal. Revista de Saúde Pública, v. 37, p. 440445, 2003.

\section{Elaine Aparecida Rocha Domingues \\ Doutora em Ciências da Saúde pela Universidade Estadual de Campinas. Coordenadora e Professora do Curso de Enfermagem da Universidade Vale do Rio Verde (UNINCOR), Três Corações, Minas Gerais. \\ Camila Regina de Souza Antenor \\ Graduanda do Curso de Enfermagem da Universidade Vale do Rio Verde (UNINCOR), Três Corações, Minas Gerais.}

Maiúme Roana Ferreira de Carvalho

Mestre em Ciências Aplicadas à Saúde. Professora do Curso de Enfermagem da Universidade Vale do Rio Verde (UNINCOR), Três Corações, Minas Gerais.

Aline Ferreira Souza de Carvalho

Doutora em Ciências Veterinárias. Professora do Curso de Medicina Veterinária da Universidade Vale do Rio Verde (UNINCOR), Três Corações, Minas Gerais. 